\title{
Plaque and Stent Artifact Reduction in Subtraction CT Angiography Using Nonrigid Registration and a Volume Penalty
}

\author{
Dirk Loeckx ${ }^{1}$, Stylianos Drisis ${ }^{2}$, Frederik Maes ${ }^{1}$, Dirk Vandermeulen ${ }^{1}$, \\ Guy Marchal ${ }^{2}$, and Paul Suetens ${ }^{1}$ \\ 1 Medical Image Computing (ESAT/PSI), Faculty of Engineering \\ 2 Department of Radiology, Faculty of Medicine, \\ University Hospital Gasthuisberg, Herestraat 49, B-3000 Leuven, Belgium \\ Dirk.Loeckx@uz.kuleuven.ac.be
}

\begin{abstract}
Computed tomography angiography (CTA) is an established tool for vessel imaging. Yet, high-intense structures in the contrast image can seriously hamper luminal visualisation. This can be solved by subtraction CTA, where a native image is subtracted from the contrast image. However, patient and organ motion limit the application of this technique. Within this paper, a fully automated intensity-based nonrigid $3 \mathrm{D}$ registration algorithm for subtraction $\mathrm{CT}$ angiography is presented, using a penalty term to avoid volume change during registration. Visual and automated validation on four clinical datasets clearly show that the algorithm strongly reduces motion artifacts in subtraction CTA. With our method, $39 \%$ to $99 \%$ of the artifacts disappear, also those caused by minimal displacement of stents or calcified plaques. This results in a better visualisation of the vessel lumen, also of the smaller vessels, allowing a faster and more accurate inspection of the whole vascular structure, especially in case of stenosis.
\end{abstract}

\section{Introduction}

Computed tomography angiography (CTA) is an established minimally invasive tool for imaging most major and also smaller vessels in the body [1]. Since its introduction more than 10 years ago, ongoing development of CT modalities resulted in shorter image acquisition time, a better spatial resolution and improved volume coverage.

New scanning protocols create an increasing amount of data, requiring a change in the way CTA studies are visualised and interpreted. Four main visualisation techniques are currently in use: multi- or curved planar reformat, maximum intensity projection (MIP), shaded-surface display and volume rendering [1]. Multiplanar or, preferably, curved planar reformat provides the most comprehensive cross-sectional luminal assessment, but extensive user interaction is required to accurately select the vessel of interest. Also, curved planar reformat can display only a single vessel at a time. MIP, shaded-surface display and volume rendering are true $3 \mathrm{D}$ visualisation methods, where the user can assess 
the whole vessel tree simultaneously. Using modern computer graphics hardware, high-resolution real-time interaction is possible. 3D methods render an in-depth view of the CT data, not only of the contrast-enhanced vessel tree, but of other structures as well. The presence of high-intense entities, like bone, heavy calcification or endoluminal stents can seriously hamper the luminal visualisation and/or require time-consuming manual editing [3].

This problem can be solved by recording a native image immediately before contrast administration. The native image is subtracted from the contrast image, resulting in a difference image that only visualises the contrast agent, which is since long the standard procedure in 2D DSA. The quality of the difference image is often deteriorated by motion-related artifacts due to patient or organ motion, especially when stents or calcified plaques are present in the vessel of interest. As proposed by several authors, this problem can be overcome by registration of the native to the contrast image. Most authors apply a rigid [45] or piecewise rigid [36] approach, which is often limited to correcting for intra-slice deformations only. However, those approaches can not remove motion artifacts caused by inter-slice motion or require prior selection and/or segmentation of the individual objects of interest.

In this paper, we present a fully automated intensity-based nonrigid 3D registration algorithm for subtraction CT angiography. A B-spline deformation mesh is used to calculate the local deformation in every voxel, using maximisation of mutual information of corresponding voxel intensities as similarity criterion 7 . By gradually increasing the number of mesh control points during optimisation, the deformation evolves from coarse to fine and becomes more and more local. A volume conservation penalty term is introduced to prevent physically impossible or improbable deformations, such as plaque shrinkage or enlargement. A similar approach was presented by Rohlfing et al. [8], but without proper validation. Also, we apply the registration to more difficult datasets containing severe artifacts near the vessel tree. Registration quality is evaluated based on a quantitative measurement of motion artifacts in the subtraction image. Both the automated measure and visual inspection consistently confirm the ability of our nonrigid registration scheme to create quasi artifact-free subtraction CT angiography images, especially in the presence of high intense structures such as bone, plaques, and stents.

\section{Methods}

\subsection{Deformation Model}

The nonrigid deformation is modelled by a B-spline deformation mesh 910]. A grid of mesh control points is positioned over the image. To model a more global deformation, the grid spacing is large, yielding a coarse mesh with few control points. A fine mesh has a small grid spacing and many control points, allowing a more local deformation. This approach allows a gradual refinement of the deformation mesh by decreasing the grid spacing. 


\subsection{Cost Function}

The proposed cost function $E_{c}=E_{s}+\omega_{p} E_{p}$ consists of a similarity measure $E_{s}$ and a penalty term $E_{p}$, using $\omega_{p}$ to modulate the influence of the penalty term. The similarity measure is the driving force behind the registration process and aims to maximise the similarity between the two images. As the contrast agent introduces local intensity differences between the images to be registered, similarity measures assuming identical intensity levels for corresponding structures, are inappropriate. Therefore, mutual information, which models the statistical dependence between the native and contrast image, is chosen 7 . The penalty term $E_{p}$ is based on the Jacobian determinant, which models local compression or expansion, and penalises volume change. The volume penalty is $E_{p}(\boldsymbol{\mu})=\frac{1}{V} \int_{V}\left(J_{\mathbf{g}}(\mathbf{r} ; \boldsymbol{\mu})-1\right)^{2} d \mathbf{r}$, with $J_{\mathbf{g}}(\mathbf{r} ; \boldsymbol{\mu})$ the Jacobian determinant at location $\mathbf{r}$ for transformation parameters $\boldsymbol{\mu}$ and $V$ the image volume.

\subsection{Algorithm}

A multiresolution optimisation algorithm is adopted [10], using 5 multiresolution stages. The algorithm gradually evolves from a coarsely sampled deformation mesh acting on downsampled images to a dense mesh at full image resolution. In stages one and two, the image is downsampled to half the original size, while stages 3 to 5 are calculated at full resolution. Initially, a B-spline mesh spacing of 64 voxels is used, that gradually decreases to 32,16 and finally 8 voxels in stages 2,4 and 5 respectively. The multiresolution approach increases processing speed by performing the initial calculations on subsampled data. Moreover, gradually decreasing the grid spacing will first recover more global deformations and progressively advance to finer deformations, thus avoiding local optima and creating a more realistic deformation field.

\subsection{Validation Measure}

Ideally, validation of medical image processing software should be performed by medical doctors on clinical applications. During the development of the algorithm, however, this is not always feasible. For a quick and reproducible evaluation of the influence of several registration settings an automated validation measure was developed. An automated measure also avoids intra-observer variations and the influence of the visualisation settings, thereby concentrating on the registration quality. The measure models image artifacts in the difference

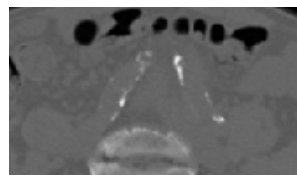

(a)

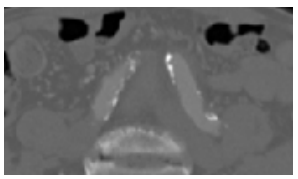

(b)

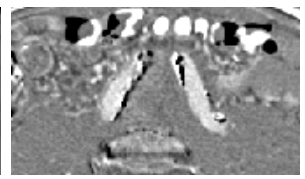

(c)

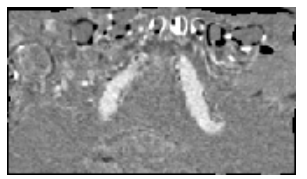

(d)

Fig. 1. Example of a (a) native and (b) contrast image, and the resulting difference image with (c) rigid and (d) nonrigid registration. In (c), the dark and bright motion artifacts are clearly visible, whereas they have almost completely disappeared in (d). 
Table 1. Overview of the intensity differences between the structures of interest in subtraction $\mathrm{CT}$ images (in $\mathrm{HU}$ )

\begin{tabular}{|c|c|c|c|c|}
\hline & ontrast & Soft Tissue & Vesse & $\begin{array}{c}\text { Plaque } \\
>300\end{array}$ \\
\hline Native & & 0 & 300 & $>300$ \\
\hline Soft Tissue & & 0 & 300 & $>300$ \\
\hline Vessel & 0 & 0 & 300 & $>300$ \\
\hline Plaque & $>300$ & $<-300$ & $<0$ & 0 \\
\hline
\end{tabular}

image. In the neighbourhood of the vessel, three important structures can be expected: soft tissue, vessel and high intense structures like calcified plaques or metal stents. An illustrative image is shown in Figure 1, and the expected intensities of these structures in the native, contrast and difference image are given in Table 1. For example, plaque voxels in the native image correctly registered to plaque voxels in the contrast image will yield an intensity difference of 0 Hounsfield units (HU) in the difference image. Plaque voxels incorrectly registered to soft-tissue in the contrast image will yield an intensity difference smaller then $-300 \mathrm{HU}$, thus causing distinct dark artifacts.

For perfectly aligned datasets, only intensities on the diagonal of the table would appear in the difference image. The accepted intensities all lie in the range $0 \rightarrow 300 \mathrm{HU}$. Due to tissue and contrast density fluctuations, noise and the partial volume effect, the actual intensity will differ from the expected one, especially along the upper limit. Therefore, we assume all voxels with intensity $<-100 \mathrm{HU}$ in the difference image to be misaligned. Misregistration also induces bright artifacts in the subtraction image, but these can not be distinguished from true enhanced vessel voxels. However, because of the volume preserving penalty, the number of voxels in the low and high intensity artifacts will be about the same. Hence, we define a region of interest (ROI) consisting of all voxels with intensity $>100 \mathrm{HU}$ in the native image (i.e. calcified regions or stents) and intensity $>-500 \mathrm{HU}$ in the post-contrast image (to exclude air) and count the fraction of voxels in this ROI with a value $<-100 \mathrm{HU}$ in the difference image (i.e. dark appearing subtraction artifacts) to measure local misregistration in high-intense regions.

\subsection{Visualisation}

Besides the computed validation measure, we also performed visual inspection of the registered images. To obtain optimal results for the volume renderer, we simultaneously applied an intensity window to the transformed native image $(-50 \rightarrow 1000 \mathrm{HU})$ and the contrast image $(100 \rightarrow 1000 \mathrm{HU})$, showing only voxels that fall inside the specified window in both images. Finally, in the difference image, the intensities are clipped to $(-50 \rightarrow 400 \mathrm{HU})$.

\section{Experiments}

The registration algorithm was applied to 4 datasets. P1 shows a healthy thoracic aorta, P2 and P3 show stented iliac arteries. P4 pictures a patient with an 
Table 2. Dimension, voxelsize and registration time for the different image datasets

\begin{tabular}{|c|c|c|c|}
\hline $\begin{array}{l}\text { Data- } \\
\text { set }\end{array}$ & $\begin{array}{l}\text { Dimensions } \\
\quad \text { (voxels) }\end{array}$ & $\begin{array}{l}\text { Voxelsize } \\
(\mathrm{mm})\end{array}$ & $\begin{array}{c}\text { Time } \\
\text { (h:m:s) }\end{array}$ \\
\hline$P 1$ & {$\left[\begin{array}{llll}91 & 136 & 411\end{array}\right]$} & $0.74^{2} \times 0.5$ & $3: 05: 20$ \\
\hline P2 & {$\left[\begin{array}{lll}158 & 187 & 261\end{array}\right]$} & $0.70^{2} \times 1.0$ & $2: 14: 08$ \\
\hline P3 & {$\left[\begin{array}{lll}114 & 177 & 144\end{array}\right]$} & $0.68^{2} \times 0.8$ & $0: 24: 24$ \\
\hline$P_{4}$ & {$\left[\begin{array}{llll}221 & 129 & 151\end{array}\right]$} & $0.74^{2} \times 1.0$ & $2: 49: 48$ \\
\hline
\end{tabular}

Table 3. Influence of the volume preserving penalty on the artifact reduction (compared to rigid registration)

\begin{tabular}{c|cccc}
$\omega_{p}$ & P1 & P2 & P3 & P4 \\
\hline 0 & $98.93 \%$ & $72.83 \%$ & $29.89 \%$ & $80.17 \%$ \\
0.01 & $98.92 \%$ & $67.94 \%$ & $27.17 \%$ & $91.29 \%$ \\
0.1 & $98.67 \%$ & $70.64 \%$ & $32.02 \%$ & $92.06 \%$ \\
1 & $\mathbf{9 9 . 0 5 \%}$ & $73.09 \%$ & $38.53 \%$ & $\mathbf{9 5 . 2 3 \%}$ \\
10 & $98.98 \%$ & $\mathbf{7 5 . 2 1 \%}$ & $\mathbf{3 9 . 4 2 \%}$ & $92.01 \%$ \\
100 & $97.51 \%$ & $74.68 \%$ & $38.56 \%$ & $94.60 \%$ \\
\hline
\end{tabular}

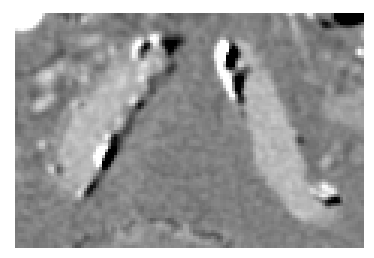

(a)

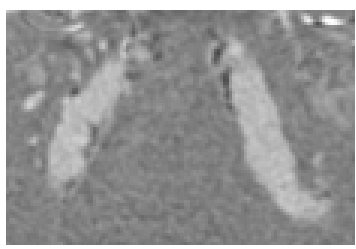

(b)

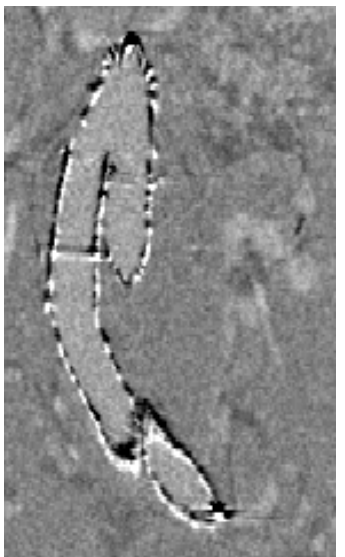

(c)

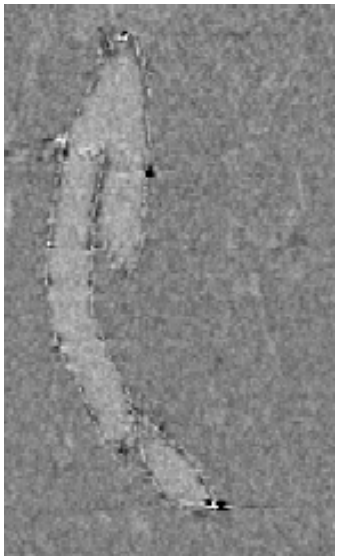

(d)

Fig. 2. (a,b) Axial slice of the highly calcified area at the aortic bifurcation of dataset P4; (c,d) sagittal slice of dataset P2 picturing a stent. These difference images show severe motion artifacts in case of rigid registration (a,c), appearing as dark and light areas near the vessel boundary. After nonrigid registration (b,d), the artifacts largely disappear.

aneurysma in the aorta abdominalis and heavy calcifications. An overview of the different datasets is given in Table 2 .

The influence of the volume penalty on the artifact reduction is shown in Table 3. The artifact reduction is expressed as the artifact fraction after non-rigid registration compared to artifact fraction after rigid registration. The minimal remaining artifact fraction is $0.21 \%, 9.04 \%, 11.56 \%$ and $0.46 \%$ for $\mathrm{P} 1, \mathrm{P} 2, \mathrm{P} 3$ and $\mathrm{P} 4$ respectively. For visual inspection, we displayed the difference image slice by slice and in a volume renderer, allowing real-time interaction with the data. This inspection confirmed the results obtained by the validation measure. Figure 2 shows some representative slices of the difference image obtained with rigid and nonrigid registration. A volume rendering of all four datasets is shown in Figure 3 . 


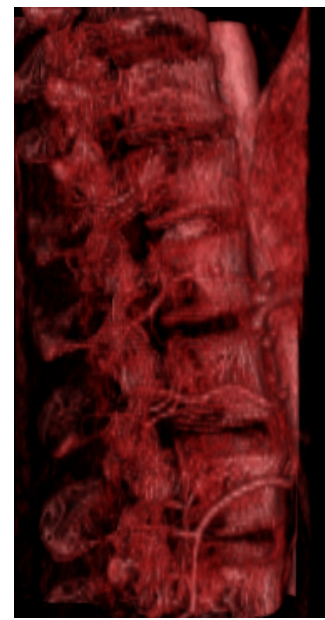

(a) P1, rigid

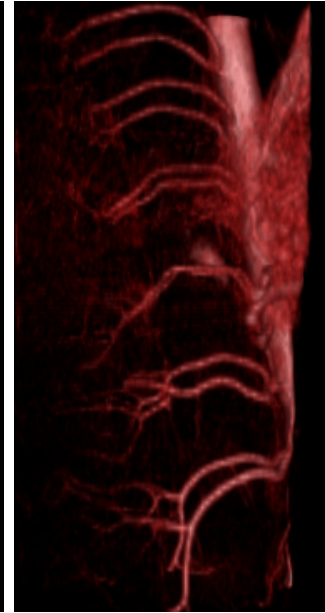

(b) P1, nonrigid

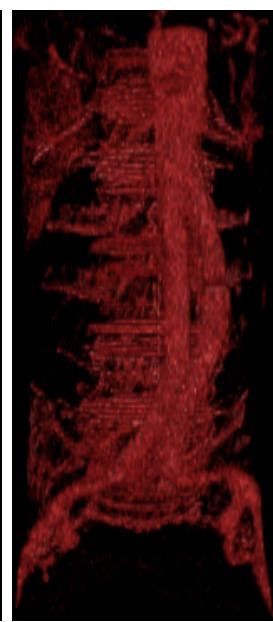

(c) P2, rigid

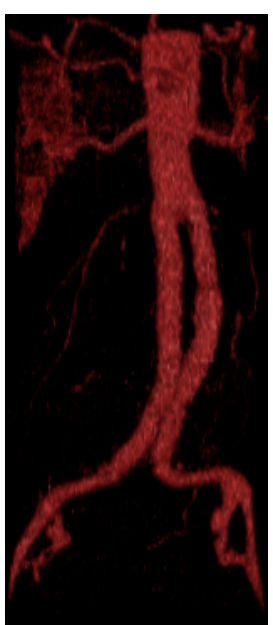

(d) P2, nonrigid

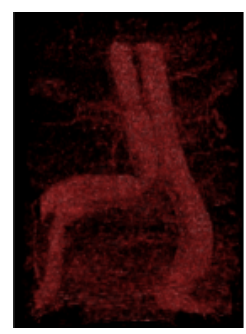

(e) P3, rigid

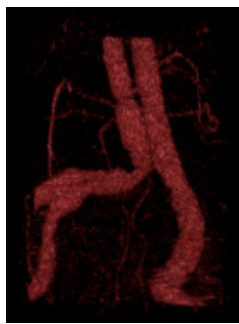

(f) P3, nonrigid

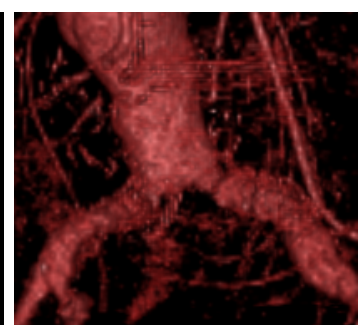

(g) P4, rigid (detail)

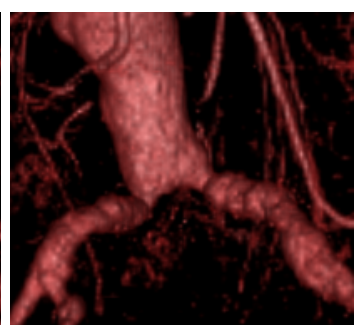

(h) P4, nonrigid (detail)

Fig. 3. Volume rendering of subtraction CT image for the different datasets using rigid and nonrigid registration. Nonrigid registration substantially reduces the motion artifacts. E.g., in (g), plaque artifacts obscure the stenosis at the bifurcation, whereas in $(\mathrm{h})$ the narrowing is clearly visible.

\section{Discussion}

\subsection{Artifacts}

It is immediately clear from Figures 2, 3 and Table 3 that nonrigid registration substantially reduces motion artifacts in the difference image. Artifacts caused by bone and calcified plaques almost disappear completely, enabling 3D visualisations, like volume rendering or MIP, to picture only the vessel lumen. This might strongly reduce reading time, as the radiologist can get a clear overview of the whole vessel. For example, in Figure $3(\mathrm{~g})$, plaque artifacts obscure the stenosis at the bifurcation, requiring a slice-by-slice or planar reformat visualisation for proper diagnosis. In Figure 3(h), the narrowing is immediately clearly visible. The artifact reduction also allows the visualisation of smaller vessels, 
which otherwise would have not been discernable from the background noise, as can be seen in Figure $3(\mathrm{~b})$.

Stent artifacts are also strongly reduced, although they are not removed completely. Due to the high attenuation difference between metallic stents and the surrounding tissue, stents will not only cause motion artifacts in the difference image, but also generate metal or streak artifacts in the native and contrast enhanced images. Especially in multi slice spiral CT, the appearance of these artifacts depends on small patient displacement and the x-ray tube starting angle 6. Therefore, they can not be removed completely, as can be seen in Figures $3(\mathrm{~d}) 3(\mathrm{f})$ and $2(\mathrm{~d})$.

\subsection{Calculation Time}

Depending on the size of the dataset, registration takes from 0.5 to 3 hours on a $2.8 \mathrm{Ghz}$ Pentium 4 processor. For diagnostic clinical use, calculations can be performed off-line and therefore timing is not critical. If emergency or interventional applications are sought, several approaches are possible to reduce the calculation time. If timing is crucial, a trade-off of registration quality for registration time can be made using less multiresolution stages. Our experience shows that the larger artifacts mostly disappear after the first two stages, allowing a reduction of the required processing time down to 5 to 30 minutes. However, the final stages are necessary to cancel out smaller artifacts. A second possibility to speed up the registration is the manual selection of a smaller region of interest, thus reducing the size of the dataset to be registered. Also, some improvements might be made to the algorithm itself. For instance, the registration could be constrained to only account for the regions in and surrounding the vessel tree by prior crude segmentation of the original images.

\subsection{Volume Preserving Penalty}

The contrast agent introduces intensity differences between the native and the contrast enhanced images. Without a volume preserving penalty, the algorithm would be tempted to reduced these differences by increasing the plaque volume, especially when using a fine mesh. However, if the volume weigh $\omega_{p}$ is too high, the mesh will be too stiff to allow registration whatsoever. Using a sub-optimal volume penalty worsens the registration and fails to correct some smaller motion artifacts. This effect was most clear in datasets P1 and P4, while datasets P2 and P3 always showed some artifacts near the stents. Table 3 indicates that the optimal value of the volume weigh $\omega_{p}$ is rather independent of the acual datasets under study. Therefore, we propose a volume weight of $\omega_{p}=1$.

\section{Conclusion}

In this paper, we showed that nonrigid registration can substantially reduce the artifacts in subtraction CT angiography, allowing for a clear 3D view of the vascular structure, also in the presence of calcified plaques and stents. We presented 
a quantitative validation of registration quality by evaluating the number of voxels that correspond to dark appearing artifacts in the difference image. Currently, the biggest concern of the algorithm is the calculation time, impeding real-time or emergency diagnostic use.

\section{Acknowledgements}

This work is part of K.U.Leuven/OF/GOA/2004/05 and FWO G.0258.02.

\section{References}

1. Napoli, A., Fleischmann, D., Chan, F., Catalano, C., Hellinger, J., Passariello, R., Rubi, G.: Computed tomography angiography: State-of-the-art imaging using multidetector-row technology. J Comput Assist Tomogr 28 (2004) S32-S45

2. Duddalwar, V.: Multislice CT angiography: a practical guide to CT angiography in vascular imaging and intervention. The Britisch Journal of Radiology 77 (2004) S27-S38

3. Beier, J., Oellinger, H., Richter, C., Fleck, E., Felix, R.: Registered image subtraction for CT-, MR- and coronary angiography. European Radiology 7 (1997) $82-89$

4. Kwon, S.M., Kim, Y.S., Kim, T.S., Ra, J.B.: Digital subtraction CT angiography based on efficient 3D registration and refinement. Comput Med Imaging Graph 28 (2004) 391-400

5. Poletti, P.A., Rosset, A., Didier, D., Bachmann, P., Verdun, F.R., Rutschmann, O., Vallee, J.P., Terrier, F., Khatchatourov, G.: Subtraction CT angiography of the lower limbs: A new technique for the evaluation of acute arterial occlusion. American Journal of Radiology 183 (2004) 1445-1448

6. van Straten, M., Venema, H.W., Streekstra, G.J., Majoie, C.B.L.M., den Heeten, G.J., Grimbergen, C.A.: Removel of bone in CT angiography of the cervical arteries by piecewise mask bone elimination. Medical Physics 31 (2004) 2924-2933

7. Maes, F., Collignon, A., Vandermeulen, D., Marchal, G., Suetens, P.: Multimodality image registration by maximization of mutual information. IEEE Trans. Med. Imag. 16 (1997) 187-198

8. Rohlfing, T., Maurer, C.J., Beier, J.: Reduction of motion artifacts in threedimensional CT-DSA using constrained adaptive multilevel free-form registration. In: Computer Assisted Radiology and Surgery (CARS 2001). (2001)

9. Rueckert, D., Frangi, A., Schnabel, J.: Automatic construction of 3D statistical deformation models using non-rigid registration. In: Proc. MICCAI. (2001) 77-84

10. Loeckx, D., Maes, F., Vandermeulen, D., Suetens, P.: Nonrigid image registration using free-form deformations with a local rigidity constraint. In: Lecture notes in computer science, vol. 3216, pp. 639-646, 2004 (Proceedings 7th international conference on medical image computing and computer-assisted intervention - MICCAI 2004, part I). (2004) 